\title{
DIRECT ANALYSIS OF AUTOMOTIVE FUELS FOR BIOETHANOL CONTENT USING RADIOCARBON ANALYSIS
}

\author{
Glenn A Norton \\ Center for Sustainable Environmental Technologies, Iowa State University, Ames, Iowa, USA. Email: nortong@iastate.edu.
}

ABSTRACT. Exploratory work was performed to investigate the feasibility of using a simplified radiometric analytical approach for determining the bioethanol content of US automotive fuels. The method involves mixing fuel samples directly with a suitable fluor. Sample preparation is extremely rapid since all conventional sample preparation steps are essentially eliminated. Results are based on the background-corrected DPM values obtained when using $10 \mathrm{~mL}$ of sample mixed with $10 \mathrm{~mL}$ of Permafluor $\mathrm{E}^{+}$. Results are also reported in terms of conventional $\mathrm{pMC}$ for some of the samples. Bioethanol from a dry-mill ethanol plant served as the analytical reference sample that represented a $100 \%$ bioethanol content. Using currentday bioethanol as a reference sample eliminates the need to correct for "bomb carbon." For 1:1 mixtures of sample and fluor, the background-corrected DPM showed a linear relationship with the bioethanol concentration, indicating that the quench correction approach was effective for the variable-quench samples. Based on the analysis of E0 (pure gasoline), E10, and "E85" from local gas stations, it appears that the method has good potential for determining the bioethanol content in commercial ethanol/gasoline blends. However, a variety of potential sources of error still require investigation in order to refine the method.

\section{INTRODUCTION}

The US Energy Policy Act of 2005 includes a renewable fuels standard (RFS) that requires increased use of renewable fuels in gasoline (Renewable Fuels Association 2005). The RFS mandates doubling the use of biorenewable ethanol and biodiesel by 2012. The primary renewable automotive fuel in the foreseeable future for the US is bioethanol. Methods are in place to test gasoline/ ethanol blends to determine the ethanol concentration in those blends, but no methods are in place to determine whether or not the ethanol is derived from biorenewable resources. With current tax incentives to use bioethanol and with the rapidly expanding production of bioethanol in the US, substituting petroleum-derived ethanol for bioethanol is currently not a significant concern. Nonetheless, there may be instances where verification of the bioethanol content of automotive fuels may be desired. In the future, it may even be desirable to spot-check automotive fuels to verify compliance with legislative requirements.

In the past few years, interest has been shown in using radiocarbon analysis to determine the biorenewable carbon component of automotive fuels. A good overview of conventional ${ }^{14} \mathrm{C}$ analytical methods that can be used to distinguish between bioethanol and petroleum-derived ethanol was recently published (Tamers 2006). The principles involved with such analyses are very similar to those used for determining the "biobased content" of commercial products for the USDA BioPreferred ${ }^{\mathrm{SM}}$ products program (US Department of Agriculture 2005; Noakes et al. 2006; Norton and Devlin 2006; Norton et al. 2007). The biobased content measurements are performed using ${ }^{14} \mathrm{C}$ analytical procedures specified in ASTM Method D6866 (ASTM International 2006).

Researchers have recently studied whether or not the biobased components in hydrocarbon fuels could be identified by using ${ }^{14} \mathrm{C}$ analyses (Dijs et al. 2006). In that study, different fuel blends were prepared by mixing (on a mass basis) bioethanol, fossil ethanol, and gasoline. The mixtures were analyzed by an unconventional liquid scintillation counting (LSC) approach in which the conversion of sample carbon to $\mathrm{CO}_{2}$ (the first step in the benzene synthesis procedure) was eliminated. Instead, the fuel blends were added directly to the fluor. The LSC analyses of the blended samples indicated that the ${ }^{14} \mathrm{C}$ activity was directly proportional to the concentration of biocarbon in each blend.

This paper also discusses the direct (no sample combustion) LSC approach for analyzing automotive fuels to determine the bioethanol concentration. As with the work by Dijs et al. (2006), the fuel sam- 
ples were mixed directly with the fluor, thereby skipping the traditional step of first converting the sample carbon to $\mathrm{CO}_{2}$. However, unlike previous published work, automotive fuels directly from the pumps at service stations were analyzed. Also, rather than working with samples on a mass basis, our samples were prepared and analyzed on a volume basis in order to relate directly to how the fuels are commercially blended. Relative to current standard practices for radiometric analysis of liquid samples, the method is inexpensive and greatly simplified, since virtually all conventional sample preparation procedures are eliminated. The work discussed in this paper is intended only to be an exploratory study to investigate the feasibility of using this approach for analyzing automotive fuels for bioethanol content. The method may not be applicable to all types of automotive fuels. However, the intent of this work is to determine whether this simplified radiometric analytical approach has potential for analyzing some types of liquid fuel samples (i.e. gasoline/ethanol blends).

\section{EXPERIMENTAL}

\section{Samples}

A sample of corn-derived ethanol was obtained from a dry-mill ethanol plant operated by Midwest Grain Processors near Lakota, Iowa, USA. That alcohol sample had a purity of $99.13 \%$, with the remainder being almost entirely water (as determined by in-house analyses at the ethanol plant). Synthetic ethanol (Aaper Alcohol \& Chemical Company, Shelbyville, KY, USA) was used for making the blank solutions for the radiometric analyses. The synthetic ethanol was produced by the catalytic conversion of ethene gas to ethanol. Since the ethene is a petrochemical, it has essentially no biobased carbon content. Accelerator mass spectrometry (AMS) analysis on the synthetic ethanol indicated that the pMC value was $<0.5 \%$, thereby confirming that this was petro-ethanol.

Fuel samples were obtained from gas stations in the vicinity of Ames, Iowa. This included E0 (unleaded gasoline with no ethanol), E10, and "E85." The fuels obtained for this study are summarized in Table 1. It must be noted that "E85" is used loosely, since the actual ethanol content varies considerably with the season. In the winter months, the ethanol concentration is decreased to improve cold weather starting. In Iowa, "E85" can contain anywhere from 70-85\% ethanol by volume, depending on the season. At the time the "E85" sample was collected from the Kum \& Go station, the "E85" actually contained 75\% ethanol, which is known as a "Class 2" mixture (US Department of Energy 2006). For the other "E85" sample, the gasoline was in a transitional phase between E70 (a "Class 3" mixture) and E75, so the ethanol content was not known definitively. However, it is certain that the fuel was either E70 or E75.

Table 1 Summary of fuels obtained from local gas stations.

\begin{tabular}{llll}
\hline Fuel & Marketing name & Brand & Octane rating \\
\hline E0 \#1 & Regular Unleaded & Unspecified & 87 \\
E0 \#2 & Premium Unleaded & Phillips 66 & 91 \\
E0 \#3 & Ultimate Unleaded & Amoco & 92 \\
E0 \#4 & Regular Unleaded & Sinclair & 87 \\
E10 \#1 & Unleaded Plus & Phillips 66 & 89 \\
E10 \#2 & "Ethanol" & Conoco & 89 \\
E10 \#3 & Amoco Silver & Amoco & 89 \\
E10 \#4 & Super Unleaded & Sinclair & 90 \\
E70-E75 & "E85" & Not specified & Not specified \\
& & (Cenex Station) & \\
E75 & "E85" & Not specified & Not specified \\
& & (Kum \& Go Station) & \\
\hline
\end{tabular}


The Amoco Ultimate Unleaded E0 fuel had virtually no color to it. All of the other E0 fuels, as well as all the E10 fuels, had a considerable yellow tint. The E85 fuels also had a yellowish tint, but it was not nearly as intense because of the high ethanol content.

In addition to the fuel samples collected at local gas stations, E10 and an E85 "standards" were prepared in-house by mixing the bioethanol with the Sinclair E0 gasoline. When preparing the standards, adjustments were made to compensate for the small water content of the bioethanol.

\section{Analytical Procedures}

All samples were analyzed using a Packard Tri-Carb 3170 TR/SL liquid scintillation counter (PerkinElmer Life Sciences, Boston, MA, USA) using QuantaSmart ${ }^{T M}$ LSC software. The fluor used in this study was Permafluor $\mathrm{E}^{+}$(PerkinElmer). Unless otherwise noted, the energy window used for analysis was $3-156 \mathrm{keV}$, and a $2-\sigma$ statistical terminator of $1.5 \%$ was applied. A dark adaptation period of $5 \mathrm{hr}$ was used prior to all analyses.

The factory-installed quench curve from the manufacturer was used to correct CPM (counts per minute) values to DPM (disintegrations per minute) values. This was performed using the instrument's "transformed spectral index of the external standard" (tSIE) feature as the quench-indicating parameter. The external standard is a ${ }^{133} \mathrm{Ba}$ source with an activity level of $1 \mathrm{Ci}$. In the tSIE quench measurement approach, the spectrum of the sample is collected in the presence and in the absence of the ${ }^{133} \mathrm{Ba}$ standard. The net spectrum for just the external standard is obtained by subtracting the sample spectrum from the combined (unknown sample plus ${ }^{133} \mathrm{Ba}$ standard) spectrum. A "reverse summation" technique is then used to transform the net spectrum into a transformed external spectrum. The amount of quench in the unknown sample is directly proportional to the maximum energy of the transformed spectrum. The tSIE value reflects that maximum energy value multiplied by the instrument calibration factor. Additional details on this approach can be found in a marketing brochure provided by the instrument manufacturer (PerkinElmer 2005).

In the direct analysis approach, the DPM/g carbon (and therefore $\mathrm{pMC}$ ) is not determined, but rather the volumetric concentrations of bioethanol in the samples are being measured. The backgroundsubtracted DPM in any given sample would theoretically decrease proportionally with decreasing bioethanol concentration. Therefore, if the background-corrected DPM in a given volume of automotive fuel is half of that observed for the same volume of bioethanol, then the bioethanol concentration in the automotive fuel would theoretically be $50 \%$. In order to verify this linear relationship (i.e. to verify that the quench indicating parameter was doing a reasonably good job at correcting for variable sample quench), a series of samples were prepared by mixing various amounts of bioethanol with the fluor. Samples of fluor containing 5-95\% (v/v) bioethanol were prepared and analyzed. These samples were counted for 800 min each.

All other analyses were performed using 1:1 mixtures of sample and fluor. Since the gasoline blends from the filling stations are mixed on a volume basis, the samples analyzed in this study were also mixed on a volume basis. The samples were prepared by pipetting $10 \mathrm{~mL}$ of sample and $10 \mathrm{~mL}$ of fluor into a 20-mL low-potassium glass scintillation vial using a Gilson Pipetman P5000 pipette. The contents of the vial were shaken prior to analysis, and all samples were completely miscible in the fluor.

A series of tests were initially performed to establish that the bioethanol being used as a reference sample had a pMC value consistent with what would be expected for bioethanol produced within the past few years. In this study, it was assumed that the current pMC value is about $107 \%$. However, it should be noted that there is some controversy on what the precise pMC value currently is. There 
may be regional variations as well as very localized variations within a given region. This is an issue that requires further investigation.

Five replicate analyses using the direct (no sample combustion) LSC procedure were performed, with a new sample preparation being performed for each analysis. The pMC values were obtained after first dividing the sample DPM by the amount of carbon theoretically present in $10.0 \mathrm{~mL}$ of ethanol. For pure ethanol, there would be $4.11 \mathrm{~g}$ of carbon in $10.0 \mathrm{~mL}$. This was calculated based on the fact that ethanol contains $52.1 \%$ by weight, and has a density of $0.789 \mathrm{~g} / \mathrm{mL}$. When taking into account the small $(0.87 \%$ ) amount of water present, this equates to $4.07 \mathrm{~g}$ of carbon in $10.0 \mathrm{~mL}$ of ethanol. For comparison, the bioethanol was also analyzed using conventional AMS procedures at a commercial testing laboratory. Results of the LSC analyses were corrected for isotopic fractionation by normalizing the data to a $\delta^{13} \mathrm{C}$ value of $-25.0 \%$. This normalization allowed for a more precise comparison between the pMC values obtained by LSC and the pMC value obtained using AMS.

Next, actual fuel samples (including the E10 and E85 "standards") were analyzed to determine the volumetric ethanol concentration in each sample. With the $2-\sigma$ statistical terminator of $1.5 \%$, the E85 and bioethanol samples were counted for roughly $300 \mathrm{~min}$, while the remaining samples were counted for 900 to $1200 \mathrm{~min}$. In this series of tests, the results were not adjusted for isotopic fractionation since 1) $\delta^{13} \mathrm{C}$ values were not obtained on every sample, and 2) it was determined that corrections for isotopic fractionation did not affect the final results when reported to the nearest $1 \%$ ethanol concentration. For informational purposes, some of the gasoline samples (including the E10 and E85 "standards") were also analyzed by AMS at a commercial testing laboratory.

Because the samples to be analyzed were prepared in the laboratory on a volume basis, and since the fuels from the gas stations are mixed on a volume basis, it is not necessary to quantify carbon contents and obtain results in terms of DPM/g carbon. The more direct approach is to simply measure the total background-corrected DPM associated with $10 \mathrm{~mL}$ of sample (mixed with $10 \mathrm{~mL}$ of fluor). As noted earlier, the bioethanol serves as a reference point that defines the DPM associated with a sample containing only bioethanol. Assuming that the background-subtracted DPM has a linear relationship with the concentration of bioethanol in the sample, the DPM associated with each of the fuels is then a direct measure of the bioethanol concentration. Specifically, the percent bioethanol in the fuel sample is calculated as follows:

$\%$ Bioethanol in fuel $=($ measured DPM in fuel sample $)(100) /$ measured DPM in bioethanol(1)

This approach also avoids the use of oxalic acid standards. As such, no corrections are needed for "bomb carbon" since ${ }^{14} \mathrm{C}$ count rates are compared directly to a post-bomb, modern reference sample (bioethanol) rather than a pre-bomb standard. Although the ${ }^{14} \mathrm{C}$ activity associated with "bomb carbon" is declining annually, the magnitude of the annual decline is currently very slight. The amount of change in the ${ }^{14} \mathrm{C}$ bomb carbon activity over several years is not currently sufficient to significantly affect the analytical results when reported to the nearest $1 \%$ bioethanol concentration. However, it may be necessary to obtain a new bioethanol reference sample every few years in order to ensure that the bomb carbon activity in the reference sample is close to that of the ethanol component of the retail fuels.

\section{RESULTS AND DISCUSSION}

\section{Sample-to-Fluor Ratio}

For the tests with various concentrations of bioethanol mixed directly with the fluor, the counting uncertainties (2- $\sigma$ percentages) were $1.5 \%$ or less for the gross counts in the $0-156 \mathrm{keV}$ region. 
Results of those tests are shown in Figures 1 and 2. Figure 1 shows the background-subtracted counts per minute (CPM) in the $0-156 \mathrm{keV}$ energy window, as well as the counting efficiency observed for each bioethanol concentration. The counting efficiency declined slightly as the ethanol concentration increased from about 5-70\%, and then declined rapidly with further increases in ethanol concentration. The CPM increased with increasing ethanol concentration up to about $75 \%$ ethanol. At higher ethanol concentrations, the CPM rapidly decreased with increasing ethanol content. In that "failure zone," there was not an adequate amount of fluor present.

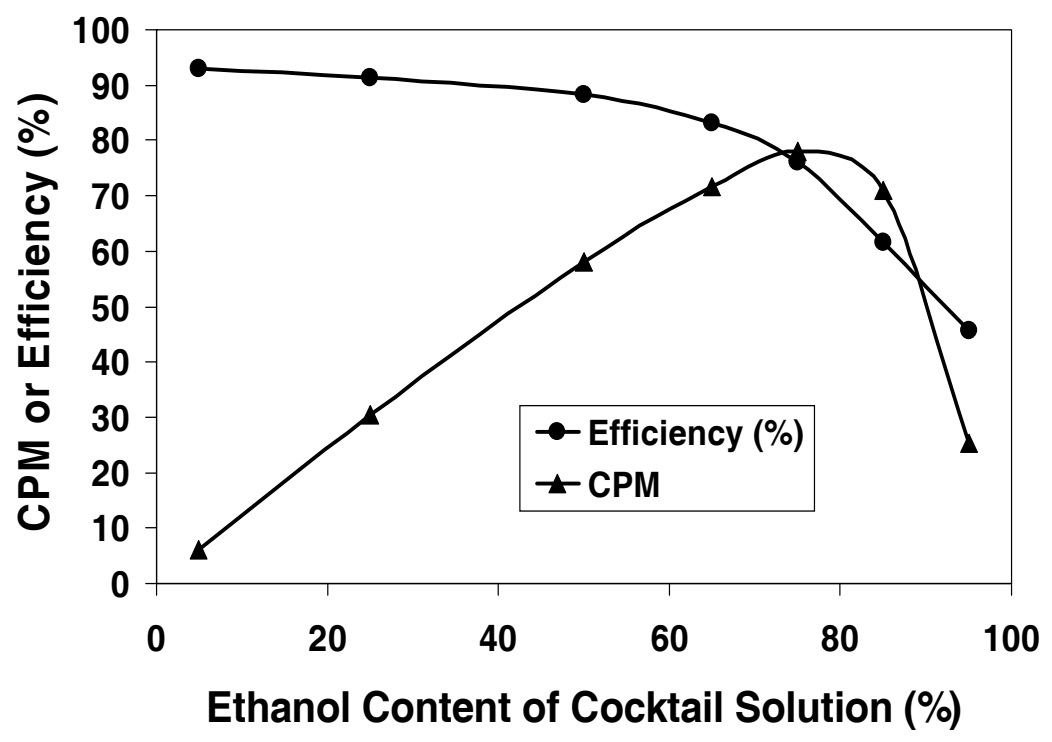

Figure 1 Background-subtracted results obtained in the $0-156 \mathrm{keV}$ region

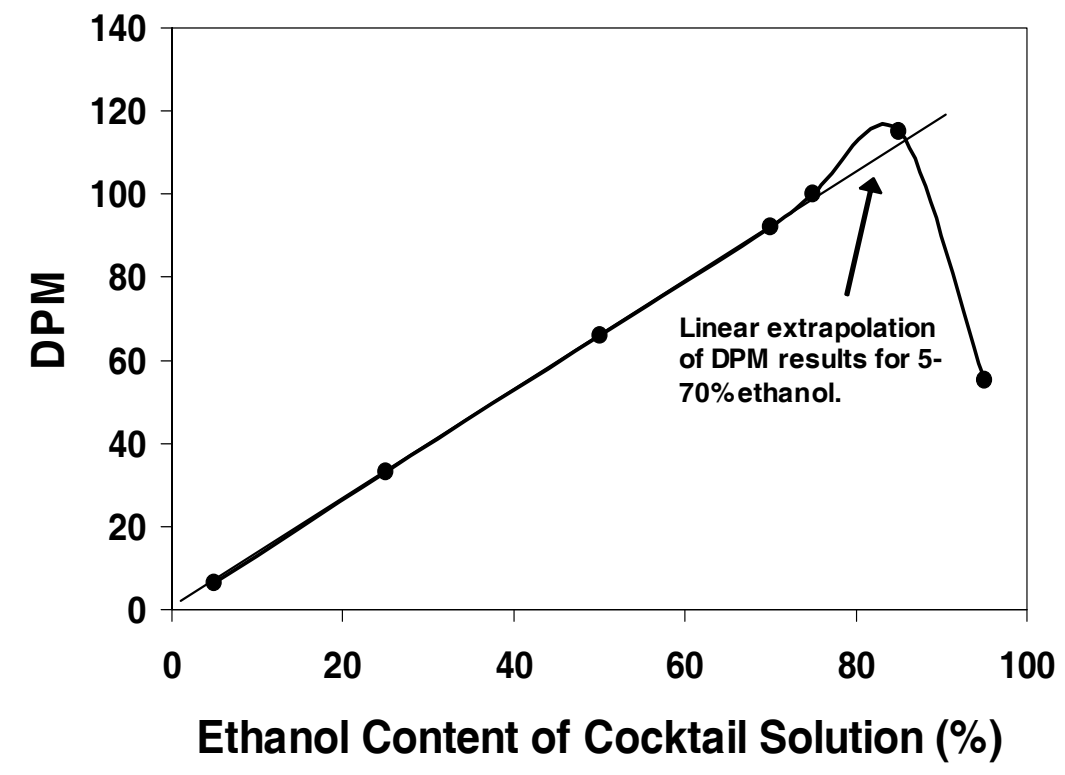

Figure 2 Background-subtracted DPM in the $0-156 \mathrm{keV}$ range as a function of the bioethanol content of the solution. 
Figure 2 shows the background-subtracted DPM results for the entire range of bioethanol concentrations that were tested. This figure also shows a linear extrapolation of results obtained for ethanol concentrations of 5-70\%. As can be seen from the plot, the background-subtracted DPM starts to deviate slightly from a linear relationship (with ethanol concentration) at $75 \%$ ethanol. The background-subtracted DPM values for ethanol concentrations of 5-70\% were highly linear, having an $R^{2}$ value of 0.9999 for the "best fit" line. This result shows that the quench correction approach worked for these variable-quench samples. Since the analyses of retail fuels used a 1:1 blend of sample and fluor, the maximum ethanol concentration in these samples was $50 \%$. This concentration corresponds to the sample that contained bioethanol as the reference sample (i.e. $10 \mathrm{~mL}$ of bioethanol and $10 \mathrm{~mL}$ of the fluor). Therefore, all samples are within the linear range of analysis.

\section{pMC Values for Bioethanol}

The AMS analysis on the bioethanol sample indicated that it had an activity level of $14.56 \mathrm{DPM} / \mathrm{g}$ carbon. This activity level corresponds to $107.4 \mathrm{pMC}$, which is the same as the pMC value that was assumed to represent modern day (last few years) corn-derived ethanol in this study. However, as noted earlier, there is some controversy on what the current pMC value truly is, and how much it might vary regionally and locally.

Five replicate tests were performed using the LSC procedure to determine the pMC value for the bioethanol. The DPM values for those samples ranged from 59.7 to 60.8 for the $4.07 \mathrm{~g}$ of carbon theoretically present in each sample (taking into account the small amount of water present). Based on those analyses, an average pMC value of 106.3 was obtained when using DPM/g carbon data normalized to a $\delta^{13} \mathrm{C}$ value of $-25.0 \%$. This constitutes a deviation of $1 \%$ (relative) from the $\mathrm{pMC}$ value determined by AMS. For each of the 5 individual pMC values that were obtained by LSC, the deviation from the pMC value obtained by AMS ranged from $0.1 \%$ to $2.0 \%$ (relative). A significant portion of this variability is believed to be due to pipetting errors.

\section{Analysis of Fuel Samples}

As noted earlier, some of the fuel samples were analyzed by AMS for informational purposes. Results of those analyses are shown in Table 2. However, it must be emphasized that the AMS data are based on the conventional approach of using DPM/g carbon. Therefore, the data do not relate directly to the bioethanol concentration on a volumetric basis because the 2 primary fuel components, gasoline and ethanol, have different concentrations of carbon and different fuel densities. Therefore, as the percent ethanol in the fuel blend changes, the total carbon in the sample changes, and the percentage of the total carbon associated with each fuel component changes.

Table 2 AMS results obtained on selected samples.

\begin{tabular}{llc}
\hline Sample & $\begin{array}{l}\delta^{13} \mathrm{C} \\
(\%)\end{array}$ & pMC \\
\hline E0 \#3 & -28.1 & 0.15 \\
E0 \#4 & -27.9 & 0.38 \\
E10 \#3 & -26.3 & 7.7 \\
E10 \#4 & -26.6 & 7.2 \\
E75 "Standard" & -16.0 & 70.8 \\
E10 "Standard" & -13.8 & 7.8 \\
E85 "Standard & -30.6 & 0.40 \\
Petro-ethanol & -11.8 & 107.4 \\
Bioethanol & \\
\hline
\end{tabular}


In the LSC analyses, the level of quench varied significantly for all of the E0 fuels and correlated with the color intensity of the fuel. In addition to color, the chemical composition of the fuel also affects quench. Variations in concentrations of various hydrocarbon components and minor additives between fuels likely results in variable quench. The tSIE values for the E0 fuels ranged from 390 to 550 , which corresponds to counting efficiencies (in the 3-156 keV range) of $85.9 \%$ to $89.0 \%$, respectively. A slight difference in background counts between petrobased alcohols and E0 samples was also noted. While methanol and synthetic ethanol had the same count rates and virtually identical spectra from the multichannel analyzer, count rates were slightly higher than those from any of the E0 samples. In addition, the background count rates among the E0 samples showed significant (e.g. $10 \%$ ) variability between samples, which could potentially be due to traces (to a variable degree) of biocarbon in E0 fuels. Since bioethanol was the "analyte" in this work, and because of the variability in the background count rates from different E0 samples, synthetic ethanol (mixed with the fluor) was used as the solution blank for all tests. In the future, it would be beneficial to determine whether "matrix matching" between the fuel samples and the solution blanks is an important issue.

The bioethanol concentrations determined in the fuel samples by the direct LSC method (using Equation 1) are shown in Table 3. The DPM results are usually not reproducible to 2 significant figures. Therefore, the measured biofuel concentrations in the table are rounded to the nearest $1 \%$ bioethanol concentration.

Table 3 Bioethanol concentrations measured by the LSC approach and estimated by AMS.

\begin{tabular}{lcl}
\hline & \multicolumn{2}{c}{ \% Bioethanol } \\
\cline { 2 - 3 } Sample & Measured by direct LSC method & Estimated from AMS pMC data \\
\hline E0 \#1 & 0 & - \\
E0 \#2 & 0 & - \\
E0 \#3 & 0 & 0 \\
E0 \#4 & 0 & 0 \\
E10 \#1 & 9 & - \\
E10 \#2 & 10 & 10 \\
E10 \#3 & 8 & 10 \\
E10 \#4 & 11 & - \\
E70-E75 & 72 & 74 \\
E75 & 73 & 10 \\
E10 "Standard" & 10 & 88 \\
E85 "Standard" & 88 & \\
\hline
\end{tabular}

In addition to the bioethanol concentrations determined by the direct LSC method, estimations of the ethanol concentrations in the fuels based on the AMS pMC data are also shown in the table for informational purposes. For those estimations, it was assumed that gasoline has a specific gravity of 0.73 and a carbon concentration of $86 \%$ (wt). Based on those assumptions, the fuel blend that would be necessary to give the pMC value obtained by AMS was calculated. The assumptions used for those calculations are reasonably good. However, unlike the ethanol component, the density and carbon concentration of gasoline can be somewhat variable. The variability in the composition and density of gasoline probably would affect the final results by no more than several percent (absolute).

The LSC results appeared to be accurate to within about $2 \%$ (absolute). Some of this error is probably due to pipetting errors related to the high volatility and low viscosity of gasoline. It is likely that the accuracy can be improved by selecting a pipette designed for highly volatile, low viscosity liq- 
uids. It is also possible that the factory-installed quench curve may not be perfectly matched to these types of samples. However, for the purposes of this exploratory work, the factory-installed quench curve was adequate for assessing the viability of using the direct LSC method for analyzing blends of gasoline and bioethanol.

In the LSC analyses, the level of quench increased with higher concentrations of bioethanol in the fuel. There were some concerns about optical and chemical quenching, and whether or not the factory-installed chemical quench curve could adequately perform the necessary corrections in these variable-quench samples. However, based on the results obtained to date, these concerns do not appear to be a significant issue for gasoline samples when using the equipment utilized in this work. Although the samples analyzed in this work exhibited varying degrees of color, optical quench would be of much greater concern for samples having a stronger color intensity.

As noted earlier, ethanol has a fixed carbon concentration, while the carbon concentration of gasoline can be somewhat variable. Since the direct LSC analyses are based on DPM per unit volume of fuel (and not on DPM/g carbon), variability in the carbon content of the petroleum-based components of the fuels is not an issue. Any variability in the carbon content of the petroleum-based components is inconsequential since those components have no ${ }^{14} \mathrm{C}$ signature.

\section{CONCLUSIONS}

Based on the results obtained in this study, the direct (no sample combustions) LSC approach appears to be promising as a greatly simplified radiometric method for determining the concentration of bioethanol in automotive fuels. Because sample preparation is all but eliminated (taking only a few minutes as opposed to hours), preparation is extremely rapid and requires little operator skill relative to conventional ${ }^{14} \mathrm{C}$ analytical approaches. With these steps eliminated, the cost per analysis can be drastically reduced (potentially by as much as $90 \%$ ). Although the results obtained to date are very encouraging, additional analyses should be performed to ensure that the method is applicable to a wider variety of automotive fuels. Also, it is clear that additional efforts are needed to address some of the areas of uncertainty. Some areas that would warrant additional effort include 1) reducing the magnitude of suspected pipetting errors, 2) determining whether the quench curve requires slight modifications in view of the sample matrices involved, and 3) addressing the CPM variability observed in the E0 samples. Also, studies are needed to help define the possible localized and regional variability in pMC values for bioethanol used as a reference sample. This work applies to fuel blends containing only bioethanol and gasoline. Modifications in the procedures would be required if there were more than one source of biocarbon in the fuels.

\section{ACKNOWLEDGMENTS}

This work was supported by a grant from ConocoPhillips.

\section{REFERENCES}

ASTM International. 2006. Method D 6866-06: Determining the biobased content of natural range materials using radiocarbon and isotope ratio mass spectrometry analysis. ASTM International, West Conshohocken, PA, USA.

Dijs IJ, van der Windt E, Kaihola L, Van der Borg K. 2006. Quantitative determination by ${ }^{14} \mathrm{C}$ analysis of the biological component in fuels. Radiocarbon 48(3): 315-23.

Noakes J, Norton G, Culp R, Nigam M, Dvoracek D.
2006. A comparison of analytical methods for the cer-
tification of biobased products. In: Chałupnik S,
Schönhofer F, Noakes J, editors. LSC 2005: Advances
in Liquid Scintillation Spectrometry. Proceedings of
the 2005 International Liquid Scintillation Confer-
ence, Katowice, Poland, 17-21 October 2005. Tuc-
son: Radiocarbon. p 259-71. Norton GA, Devlin SL. 2006. Determining the modern 
carbon content of biobased products using radiocarbon analysis. Bioresource Technology 97(16):208490.

Norton GA, Hood DG, Devlin SL. 2007. Accuracy of radioanalytical procedures used to determine the biobased content of manufactured products. Bioresource Technology 98(5):1052-6.

PerkinElmer. 2005. Features and benefits guide for TriCarb liquid scintillation analyzers [WWW document]. Available at http://las.perkinelmer.com/Content/Manuals/GDE_TriCarbFeatures.pdf.

Renewable Fuels Association. 2005. Federal regulations: renewable fuels standard [WWW document]. http:// www.ethanolrfa.org/policy/regulations/federal/standard. Accessed 15 April 2007.

Tamers M. 2006. Distinguishing between 'bio-ethanol' and petroleum ethanol. Ethanol Producer Magazine June 2006:106-9.

US Department of Agriculture. 2005. Guidelines for designating biobased products for federal procurement. Federal Register 70(7):1792-812.

US Department of Energy. 2006. Handbook for Handling, Storing, and Dispensing E85. July 2006, DOE/ GO-102006-2343. Available at http://www.afdc.energy.gov/afdc/pdfs/40243.pdf. 